\title{
Stochastic orders to approach investments in condor financial derivatives
}

\author{
M.C. López-Díaz • M. López-Díaz • S.
}

Martínez-Fernández

Received: date / Accepted: date

\begin{abstract}
The comparison of investments is a key research topic in mathematical finance. Financial derivatives are popular tools for economic investments. A common financial derivative is the so-called condor derivative. A new mathematical framework for the comparison of investments in condor derivatives is introduced in this manuscript. That model is based on the theory of stochastic orders. Namely, a new family of stochastic orders to approach such comparison problems is introduced. That family is analyzed in detail providing characterizations of the new orders, properties and connections with other stochastic orderings. Results which permit to compare condor derivatives, when the prices of the underlying assets follow Brownian movements, or geometric Brownian movements, are developed. Moreover, an analysis with the DOWJONES and EUROSTOXX indexes shows how to use the new stochastic orders to compare investments in condor derivatives based on those indexes. On the other hand, it is shown how well-known stochastic orders can be applied to compare investments in other financial derivatives, like future derivatives, bull call spreads, call options or long straddle derivatives.
\end{abstract}

Keywords Call option · Condor derivative · (Geometric) Brownian movement · Increasing concave order · Put option · Stochastic order

The authors are indebted to the Spanish Ministry of Science and Innovation and to Principado de Asturias, since this research is financed by Grants MTM2013-45588-C3-1-P, MTM2015-63971-P, FC-15-GRUPIN14-101 and FC-15-GRUPIN14-142.

M.C. López-Díaz

Departamento de Matemáticas, Universidad de Oviedo. C/Calvo Sotelo s/n. E-33007 Oviedo, Spain. E-mail: cld@uniovi.es

M. López-Díaz

Departamento de Estadística e I.O. y D.M., Universidad de Oviedo. C/Calvo Sotelo s/n. E-33007 Oviedo, Spain. Tel.: +34-985103362 Fax: +34-985103354 E-mail: mld@uniovi.es

S. Martínez-Fernández

Unidad de Modelos de Riesgos, Liberbank. Plaza de La Escandalera, 2. E-33003 Oviedo, Spain. E-mail: smartinezf@liberbank.es 
Mathematics Subject Classification (2000) 60E15 $62 \mathrm{P} 05$

\section{Introduction}

Modern economy cannot be understood without the concept of financial derivative. A financial derivative is a financial contract between (at least) two parties having a value which is derived from an underlying asset (like an index, an interest rate, a commodity, a stock, bonds, currencies, other derivatives, etc.) The value of a derivative is based on the future price of the underlying asset. Derivatives can be used for multiple purposes like insuring against price movements, fluctuations in stock, changes in foreign exchange rates, changes in interest rates, increasing exposure to price movements for speculation, or for instance, getting access to other assets or markets. An investor purchases a financial derivative when he believes that the future price of the asset will be substantially different from the expected price held by the other party of the contract, and tries to benefit from that. For an introduction to the field of financial derivatives, the reader is referred for instance to the books by Dixit and Pindyck (1994), Kolb and Overdahl (2002), Hunt and Kennedy (2004), Cohen (2005), Jarrow and Chatterjea (2013) and Hull (2015).

One of the most commonly used derivatives is the so-called option, which plays an essential role in finance. An option derivative gives to its buyer the right, but not the obligation, to buy (call option) or sell (put option) an asset at an agreed-upon price (strike or exercise price) on a specific date (exercise date or expiration date) or during a certain period of time, paying for this right a premium. Throughout the paper we will consider options with an expiration date (European options) instead of options which can be exercised at any time of a period (American options). The purchaser profits from a call option when the underlying asset increases in price at the expiration date. The purchaser of a put option believes that the underlying asset will drop below the exercise price before the expiration date, and so he profits from a put option when the price of the asset decreases. If $x$ is the unit price of the underlying asset at the exercise date, $p$ is the unit exercise price considered in the contract and $k$ is the unit premium, the profit per unit of the underlying asset of the purchaser of the call option is $(x-p)_{+}-k$, where the subscript + stands for the positive part of a real number. Namely, if $x>p$, the purchaser of the call option will exercise the right of the option, and so his unit benefit will be $(x-p)-k$. On the contrary, if $x<p$, the purchaser will not exercise the right, his unit loss being the unit premium $k$. The unit profit of the seller of the call option is $k-(x-p)_{+}$. In the same way, the profits per unit of the underlying asset of the purchaser and the seller of a put option, are $(p-x)_{+}-k$ and $k-(p-x)_{+}$ respectively.

Some derivatives are defined by means of a mixture of other financial derivatives. That is the case of the condor financial derivative. This can be designed as follows. Four contracts on the same asset and same expiration date are bought or sold at four different strike prices. Namely, the condor 
derivative is the result of the purchase of a call option and a put option with unit exercises prices $p_{3}$ and $p_{2}$ and unit premiums $k_{3}$ and $k_{2}$ respectively, where $p_{2}<p_{3}$, and the sale of a call option and a put option with unit exercise prices $p_{4}$ and $p_{1}$ and unit premiums $k_{4}$ and $k_{1}$ respectively, with $p_{1}<p_{2}$ and $p_{3}<p_{4}$. If $x$ stands for the unit price of the underlying asset at the expiration date, then the benefit of the condor derivative is $-\left(p_{1}-x\right)_{+}+\left(p_{2}-x\right)_{+}+\left(x-p_{3}\right)_{+}-\left(x-p_{4}\right)_{+}+k_{1}-k_{2}-k_{3}+k_{4}$. Observe that a condor derivative has limited risk. Its maximum loss is reached when $x$ lies between $p_{2}$ and $p_{3}$, that loss given by the corresponding premiums, that is, $k_{1}-k_{2}-k_{3}+k_{4}$. The largest return of a condor derivative is also limited and it arises when $x$ is at least $p_{4}$ or at most $p_{1}$. An investor purchases a condor derivative when he estimates that the price of the underlying asset will not be close to the interval $\left[p_{2}, p_{3}\right]$ at the expiration date.

Investors frequently have the possibility to choose between two condor derivatives of different assets under the same contractual conditions, that is, with same exercise prices, premiums and expiration dates, to make an investment. That is very common when underlying assets are different kinds of an agricultural product, for instance, the case of citrus fruits. Some varieties of oranges have the same prices at the beginning of the season, but their prices change during the sowing time (depending on the humidity, the land, the rain, the temperature, stocks, etc.), and so they are different at the end of the harvest.

The main aim of this manuscript is to introduce a mathematical model to compare investments in condor derivatives under that framework, and study and analyze in detail such a model. The paper has the following structure. Section 2 is devoted to present the concepts, terminology and notations that we need in the manuscript. In Section 3, motivated by the problem described above, a new family of stochastic orders is introduced to compare investments in condor financial derivatives. Main results of the article are included in Section 4, characterizations of the stochastic orders, properties and connections with other orders are developed. In Section 5, we consider a financial application of the proposed mathematical model. Namely, we obtain conditions to order prices of assets in condor stochastic orders, when those prices follow Brownian motions with drifts or the Black-Scholes model. Section 6 shows an empirical analysis of condor stochastic orders for the DOWJONES and the EUROSTOXX indexes. In Section 7, we show how "classic" stochastic orders can be used to compare investments in other financial derivatives. Section 8 summarizes the main conclusions of the manuscript. An appendix at the end of the manuscript contains the proofs of the mathematical results.

\section{Preliminaries}

Roughly speaking, a stochastic order tries to order probabilities in accordance with an appropriate criterion. Formally, a stochastic order is a pre-order relation on a set of probabilities associated with a measurable space. Stochastic 
orderings has been applied successfully in many fields like genetics, ecology, biology, medicine, ophthalmology, statistical physics, decision theory, queueing systems, scheduling problems, control theory, reliability theory, economy, etc. For a detailed analysis of the theory and applications of stochastic orderings, the reader is referred for instance to the books by Müller and Stoyan (2002), Shaked and Shanthikumar (2007) and Belzunce et al. (2016).

In this manuscript we will introduce a family of stochastic orders on the set of probabilities associated with the measurable space $(\mathbb{R}, \mathcal{B})$, where $\mathcal{B}$ stands for the usual Borel $\sigma$-algebra on $\mathbb{R}$.

If $\preceq$ denotes a stochastic order on the set of probabilities on $(\mathbb{R}, \mathcal{B})$, and $X$ and $Y$ are two random variables, $X \preceq Y$ will mean that $P_{X} \preceq P_{Y}, P_{X}$ and $P_{Y}$ being the distributions induced by $X$ and $Y$ respectively.

Given a random variable $X, F_{X}$ will denote its distribution function, $E X$ its expected value and $\operatorname{Var} X$ its variance. If $X$ is continuous, $f_{X}$ will denote its probability density function. The integrated survival function of a random variable with finite mean is the mapping $\pi_{X}: \mathbb{R} \rightarrow \mathbb{R}$ with $\pi_{X}(t)=E(X-t)_{+}$ for any $t \in \mathbb{R}$.

The following stochastic orderings will appear throughout the paper. Let $X$ and $Y$ be two random variables,

i) $X$ is said to be smaller than $Y$ in the usual stochastic ordering if $E(f(X)) \leq E(f(Y))$ for all increasing mappings $f: \mathbb{R} \rightarrow \mathbb{R}$ such that the above expectations exist, equivalently, if $F_{X} \geq F_{Y}$. It will be denoted by $X \preceq_{s t} Y$;

ii) $X$ is said to be smaller than $Y$ in the (increasing) convex order if $E(f(X)) \leq E(f(Y))$ for all (increasing) convex mappings $f: \mathbb{R} \rightarrow \mathbb{R}$ such that the above expectations exist. It will be denoted by $\left(X \preceq_{i c x} Y\right) X \preceq_{c x} Y$;

iii) $X$ is said to be smaller than $Y$ in the (increasing) concave order if $E(f(X)) \leq E(f(Y))$ for all (increasing) concave mappings $f: \mathbb{R} \rightarrow \mathbb{R}$ such that the above expectations exist. It will be denoted by $\left(X \preceq_{i c v} Y\right) X \preceq_{c v} Y$;

$i v$ ) when $X$ and $Y$ have symmetric distributions with respect to $\mu_{X}$ and $\mu_{Y}$ respectively, it is said that $X$ is smaller than $Y$ in the peakness order if $\left|X-\mu_{X}\right| \preceq_{s t}\left|Y-\mu_{Y}\right|$. It will be denoted by $X \preceq_{\text {peak }} Y$ (see Birnbaum (1948) and Bickel and Lehmann (1976));

v) $X$ is said to be less dispersive than $Y$ in the weak dispersion ordering if $\left|X-X^{\prime}\right| \preceq_{s t}\left|Y-Y^{\prime}\right|$, where $X^{\prime}$ and $Y^{\prime}$ are independent copies of $X$ and $Y$ respectively. This relation will be denoted by $X \preceq_{w} Y$ (see Giovagnoli and Wynn (1995));

$v i)$ it will be said that $X$ is smaller than $Y$ in the bidirectional ordering, denoted by $X \preceq_{b d} Y$, if $X_{+} \preceq_{s t} Y_{+}$and $X_{-} \preceq_{s t} Y_{-}$hold simultaneously (see Müller (1998) and López-Díaz (2010)).

A stochastic order $\preceq$ on the set of probabilities associated with $(\mathbb{R}, \mathcal{B})$ is said to be integral, if there exists a class of measurable mappings satisfying that $P \preceq Q$ when $\int_{\mathbb{R}} f d P \leq \int_{\mathbb{R}} f d Q$ for all $f$ in that class, such that the above integrals exist. That class of mappings is said to be a generator of the order. It is well-known that there could be different generators of the same stochastic order (see Müller (1997)). 
Given $X$ and $Y$ random variables, $X \sim_{s t} Y$ will mean that they are equal in distribution. If $P$ is a probability on $(\mathbb{R}, \mathcal{B})$ and $T: \mathbb{R} \rightarrow \mathbb{R}$ is a measurable mapping, $P \circ T^{-1}$ will denote the probability given by $P \circ T^{-1}(B)=P\left(T^{-1}(B)\right)$ for any $B \in \mathcal{B}$. If $A$ is a subset of $\mathbb{R}, I_{A}$ will stand for the indicator function of $A$. Let $a \in \mathbb{R}, a_{+}$will stand for $\max \{a, 0\}$, and $a_{-}$for $\max \{-a, 0\}$. On the other hand [.] will denote the integer part. Throughout the paper, increasing will mean non-decreasing and decreasing will mean non-increasing.

\section{The new family of stochastic orders}

In this section we introduce the new family of stochastic orders which arises from the analysis of the problem described in the Introduction of the manuscript.

Let $k$ stands for $k_{1}-k_{2}-k_{3}+k_{4}$. Let $p_{1}<p_{2}<p_{3}<p_{4}$. Define the mapping $f_{p_{1}, p_{2}, p_{3}, p_{4}, k}: \mathbb{R} \rightarrow \mathbb{R}$ with $f_{p_{1}, p_{2}, p_{3}, p_{4}, k}(x)=-\left(p_{1}-x\right)_{+}+\left(p_{2}-\right.$ $x)_{+}+\left(x-p_{3}\right)_{+}-\left(x-p_{4}\right)_{+}+k$, that is, for any $x \in \mathbb{R}$,

$$
f_{p_{1}, p_{2}, p_{3}, p_{4}, k}(x)= \begin{cases}p_{2}-p_{1}+k & \text { if } x \in\left(-\infty, p_{1}\right], \\ p_{2}-x+k & \text { if } x \in\left(p_{1}, p_{2}\right], \\ k & \text { if } x \in\left(p_{2}, p_{3}\right], \\ x-p_{3}+k & \text { if } x \in\left(p_{3}, p_{4}\right], \\ p_{4}-p_{3}+k & \text { if } x \in\left(p_{4}, \infty\right),\end{cases}
$$

see Figure 1 for its graphical representation.

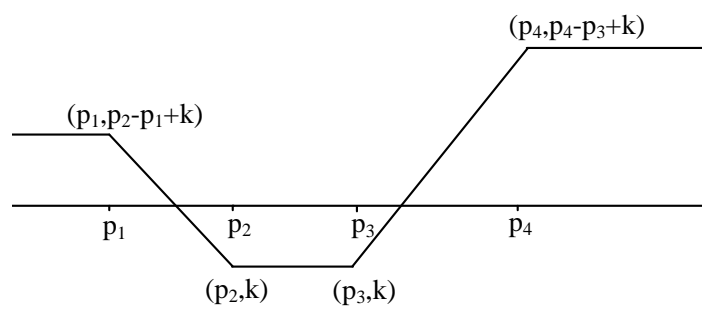

Fig 1 Graphical representation of a return of a condor derivative, $p_{1}$ unit price of the put sale, $p_{2}$ unit price of the put purchase, $p_{3}$ unit price of the call purchase, $p_{4}$ unit price of the call sale, $k$ resulting unit premium

Given $p_{2}, p_{3} \in \mathbb{R}$ with $p_{2}<p_{3}$, let $\mathcal{F}^{p_{2}, p_{3}}=\left\{f_{p_{1}, p_{2}, p_{3}, p_{4}, k} \mid p_{1}<p_{2}, p_{3}<\right.$ $\left.p_{4}, k \in \mathbb{R}\right\}$, and let $\mathcal{F}_{0}^{p_{2}, p_{3}}$ stand for the class of mappings $\left\{f_{p_{1}, p_{2}, p_{3}, p_{4}, k} \in\right.$ $\left.\mathcal{F}^{p_{2}, p_{3}} \mid k=0\right\}$. By $f_{p_{2}, p_{2}, p_{3}, p_{4}, k}$ we will denote the mapping resulting from taking $p_{1}=p_{2}$ in formula (1), omitting in its definition the case $x \in\left(p_{1}, p_{2}\right]$. In a similar way we define $f_{p_{1}, p_{2}, p_{3}, p_{3}, k}$ and $f_{p_{2}, p_{2}, p_{3}, p_{3}, k}$.

Let $X$ and $Y$ be the random variables associated with the unit prices at the expiration date of the underlying assets of two condor derivatives under the same contractual conditions. How is possible to compare both investments? That is the main aim of this paper. We introduce the following family of stochastic orders to approach this question. 
Definition 1 Let $X$ and $Y$ be random variables and let $p_{2}, p_{3} \in \mathbb{R}$ with $p_{2}<p_{3}$. It will be said that $X$ is less than $Y$ in the condor stochastic order for the purchase prices $p_{2}$ and $p_{3}$, if $E(f(X)) \leq E(f(Y))$ for any $f \in \mathcal{F}^{p_{2}, p_{3}}$. Such a condition will be denoted by $X \preceq_{\text {con }}^{p_{2}, p_{3}} Y$.

All the mappings of $\mathcal{F}^{p_{2}, p_{3}}$ are bounded, and so for any random variables $X$ and $Y$ and any $f \in \mathcal{F}^{p_{2}, p_{3}}$, the expected values $E(f(X))$ and $E(f(Y))$ exist.

Let us clarify the meaning of the new family of stochastic orders. Investors frequently have the opportunity to design condor derivatives where prices $p_{2}$ and $p_{3}$ are fixed because of previous purchases, playing adequately with the sale prices $p_{1}$ and $p_{4}$ to try to maximize their benefits. Under this framework and if they have the possibility to buy two condor derivatives under the same contractual conditions, which one is preferred for an investment? Let $X$ and $Y$ denote the unit prices of the underlying assets at the expiration date in the above condor derivatives. The relation $X \preceq_{c o n}^{p_{2}, p_{3}} \quad Y$ means that the expected benefit of the condor derivative formed with the second asset, is greater than or equal to the expected benefit of the condor derivative with the former, whatever prices $p_{1}$ and $p_{4}$ of the call and put option sales respectively. Under those conditions, the investment in the second condor derivative is preferable to the investment in the first derivative. Anyway, in case of an investor wants to invest in a condor derivative with the first asset (that associated with $X$ ), the relation $X \preceq \preceq_{c o n}, p_{3} \quad Y$ shows that the premium paid for that must be lower than the premium of the condor derivative associated with $Y$.

Condor stochastic orders permit to compare investments in condor derivatives under the above framework. It is worth mentioning that it is possible to compare investments in common financial derivatives by means of well-known stochastic orders, as we prove in Section 7 of the manuscript.

\section{Main results on the order}

In this section we develop the main results on the new stochastic orders. Namely, we state characterization results for the orders, derive consequences and properties, and analyze connections with other stochastic orders.

\subsection{Characterizations}

In this subsection different characterizations results of the new family of stochastic orders are proved.

We state some technical results which will be applied afterwards.

It is clear that the order $\preceq_{c o n}^{p_{2}, p_{3}}$ is integral, and the class $\mathcal{F}^{p_{2}, p_{3}}$ is a generator of that order. The following result provides a simpler generator of the stochastic order.

Lemma 1 The class of mappings $\mathcal{F}_{0}^{p_{2}, p_{3}}$ is a generator of the order $\preceq_{c o n}^{p_{2}, p_{3}}$. 
The following lemma will be useful to prove characterization results of the new family of stochastic orders.

Lemma 2 Let $X$ and $Y$ be random variables with $X \preceq_{p_{2}, p_{3}} Y$. Then

i) $E\left(f_{p_{2}, p_{2}, p_{3}, p_{4}, k}(X)\right) \leq E\left(f_{p_{2}, p_{2}, p_{3}, p_{4}, k}(Y)\right)$,

ii) $E\left(f_{p_{1}, p_{2}, p_{3}, p_{3}, k}(X)\right) \leq E\left(f_{p_{1}, p_{2}, p_{3}, p_{3}, k}(Y)\right)$ and

iii) $E\left(f_{p_{2}, p_{2}, p_{3}, p_{3}, k}(X)\right) \leq E\left(f_{p_{2}, p_{2}, p_{3}, p_{3}, k}(Y)\right)$,

for any $p_{1}, p_{2}, p_{3}, p_{4}, k \in \mathbb{R}$ with $p_{1}<p_{2}<p_{3}<p_{4}$.

Next result reads that the theoretical study of the condor stochastic order can be reduced to the analysis of the order $\preceq_{\text {con }}^{-\delta, \delta}$ with $\delta>0$ in most of the cases.

Proposition 1 Let $X$ and $Y$ be random variables, let $p_{2}, p_{3} \in \mathbb{R}$ with $p_{2}<p_{3}$ and $\mu=p_{3}-p_{2}$. Then $X \preceq_{\text {con }}^{p_{2}, p_{3}} Y$ if and only if $X-\left(p_{2}+p_{3}\right) / 2 \preceq_{\text {con }}^{-\mu / 2, \mu / 2}$ $Y-\left(p_{2}+p_{3}\right) / 2$.

We state some characterizations results of the stochastic order $\preceq_{\text {con }}^{-\delta, \delta}$ for any $\delta>0$. Characterizations of $\preceq_{c o n}^{p_{2}, p_{3}}$ with $p_{2}<p_{3}$ can be deduced by means of Proposition 1.

The first characterization will be key to derive simpler characterizations.

Proposition 2 Let $X$ and $Y$ be random variables and let $\delta>0$. Then $X \preceq$ con $Y$ if and only if

i) $E\left(X I_{(\delta, \delta+t]}(X)\right)-\delta\left(F_{X}(\delta+t)-F_{X}(\delta)\right)+t\left(1-F_{X}(\delta+t)\right) \leq$ $E\left(Y I_{(\delta, \delta+t]}(Y)\right)-\delta\left(F_{Y}(\delta+t)-F_{Y}(\delta)\right)+t\left(1-F_{Y}(\delta+t)\right)$ for any $t>0$, and

ii) $|t| F_{X}(-\delta+t)-\delta\left(F_{X}(-\delta)-F_{X}(-\delta+t)\right)+E\left(|X| I_{(-\delta+t,-\delta]}(X)\right) \leq$ $|t| F_{Y}(-\delta+t)-\delta\left(F_{Y}(-\delta)-F_{Y}(-\delta+t)\right)+E\left(|Y| I_{(-\delta+t,-\delta]}(Y)\right)$ for any $t<0$.

The above result permits to obtain another characterization of the order $\preceq_{c o n}^{-\delta, \delta}$ by means of distribution functions.

Proposition 3 Let $X$ and $Y$ be random variables and let $\delta>0$. Then $X \preceq_{\text {con }}^{-\delta, \delta}$ $Y$ if and only if

i) $\int_{\delta}^{\delta+t} F_{X}(x) d x \geq \int_{\delta}^{\delta+t} F_{Y}(x) d x$ for any $t>0$, and

ii) $\int_{-\delta+t}^{-\delta} F_{X}(x) d x \leq \int_{-\delta+t}^{-\delta} F_{Y}(x) d x$ for any $t<0$.

Another characterization of condor stochastic orders by means of the increasing concave stochastic order is proved now.

Proposition 4 Let $X$ and $Y$ be random variables and let $\delta>0$. Then $X \preceq_{\text {con }}^{-\delta, \delta}$ $Y$ if and only if $(X-\delta)_{+} \preceq_{i c v}(Y-\delta)_{+}$and $(X+\delta)_{-} \preceq_{i c v}(Y+\delta)_{-}$.

Corollary 1 Let $X$ and $Y$ be random variables and let $\delta>0$ such that $X \preceq_{\text {con }}^{-\delta, \delta} Y$. Then $E(X-\delta)_{+} \leq E(Y-\delta)_{+}$and $E(X+\delta)_{-} \leq E(Y+\delta)_{-}$. 
Proposition 5 Let $X$ and $Y$ be random variables. Let $p_{2}, p_{3} \in \mathbb{R}$ with $p_{2}<p_{3}$. Then $X \preceq$ con $p_{2}, p_{3} \quad$ if and only if $-X \preceq$ con $-p_{3},-p_{2}-Y$.

Corollary 2 Let $X$ and $Y$ be random variables and let $\delta>0$. Then $X \preceq-\delta, \delta$ $Y$ if and only if $-X \preceq$ con $-\delta, \delta$.

In spite of the above result, it is clear that the order $\preceq_{c o n}^{-\delta, \delta}$ is not sign-free.

The following results analyze characterizations of condor stochastic orders for symmetric distributions.

Proposition 6 Let $X$ and $Y$ be random variables with symmetric distributions with respect to 0 , and let $\delta>0$. Then $X \preceq$ con $Y$ if and only if $|X| \preceq \underset{c o n}{-\delta, \delta}|Y|$.

Corollary 3 Let $X$ and $Y$ be random variables with symmetric distributions with respect to 0 , and let $\delta>0$. Then $X \preceq \preceq_{\text {con }}^{-\delta, \delta} Y$ if and only if $(|X|-\delta)_{+} \preceq$ icv $(|Y|-\delta)_{+}$.

\subsection{Relevant properties}

This subsection is devoted to the study of important properties of condor stochastic orders.

It can be seen that condor stochastic orders are not antisymmetric. However, the following result shows that two random variables equal in a condor stochastic order have the same distribution in their tails.

Proposition 7 Let $X$ and $Y$ be random variables such that $X \preceq$ con $Y$ and $Y \preceq \preceq_{\text {con }}^{-\delta, \delta} X$ with $\delta>0$. Then $F_{X}(t)=F_{Y}(t)$ for any $t \in(-\infty,-\delta] \cup[\delta,+\infty)$.

We recall the definition of the number of sign changes of a function. Let $g: \mathbb{R} \rightarrow \mathbb{R}$ be a mapping. The number of sign changes of $g$ is given by $S^{-}(g)=\sup _{\left\{x_{1}<x_{2}<\cdots<x_{n}, x_{i} \in \mathbb{R}, n \in \mathbb{N}\right\}} S^{-}\left(g\left(x_{1}\right), g\left(x_{2}\right), \ldots, g\left(x_{n}\right)\right)$, where $S^{-}\left(y_{1}, \ldots, y_{n}\right)$ is the number of sign changes of the tuple $\left(y_{1}, \ldots, y_{n}\right) \in \mathbb{R}^{n}$, where zero values are discarded. By means of that concept, we can state conditions which lead to the condor stochastic order.

Proposition 8 Let $X$ and $Y$ be continuous random variables with symmetric distributions with respect to 0 . Let $\delta>0$. If $S^{-}\left(f_{Y}-f_{X}\right)=2$ and the sign sequence is,,+-+ , then $X \preceq \preceq_{\text {con }}^{-\delta, \delta} Y$.

Given $X_{1}, X_{2}, \ldots, X_{n}$ a random sample drawn from $X, X_{(1)}$ will stand for the random variable $\min \left\{X_{1}, X_{2}, \ldots, X_{n}\right\}$, and $X_{(n)}$ for $\max \left\{X_{1}, X_{2}, \ldots, X_{n}\right\}$.

Next result provides conditions to conclude the order $\preceq_{c o n}^{-\delta, \delta}$ for random variables, and for the maximum and the minimum of random samples of those variables. 
Proposition 9 Let $X$ and $Y$ be random variables. Let $X_{1}, X_{2}, \ldots, X_{n}$ and $Y_{1}, Y_{2}, \ldots, Y_{n}$ be random samples drawn from $X$ and $Y$ respectively. If $S^{-}\left(F_{Y}-\right.$ $\left.F_{X}\right) \leq 1$, and the sequence of signs is,-+ when $S^{-}\left(F_{Y}-F_{X}\right)=1$, we have that:

i) if $E(X-\delta)_{+} \leq E(Y-\delta)_{+}$and $E(X+\delta)_{-} \leq E(Y+\delta)_{-}$, then $X \preceq \preceq_{\text {con }}^{-\delta, \delta} Y$,

ii) if $E\left(X_{(n)}-\delta\right)_{+} \leq E\left(Y_{(n)}-\delta\right)_{+}$and $E\left(X_{(n)}+\delta\right)_{-} \leq E\left(Y_{(n)}+\delta\right)_{-}$, then $X_{(n)} \preceq{ }_{\text {con }}^{-\delta, \delta} Y_{(n)}$,

iii) if $E\left(X_{(1)}-\delta\right)_{+} \leq E\left(Y_{(1)}-\delta\right)_{+}$and $E\left(X_{(1)}+\delta\right)_{-} \leq E\left(Y_{(1)}+\delta\right)_{-}$, then $X_{(1)} \preceq_{\operatorname{con}}^{-\delta, \delta} Y_{(1)}$.

Proposition 10 Let $X$ be a random variable and let $h_{1}, h_{2}: \mathbb{R} \rightarrow \mathbb{R}$ be measurable mappings such that $0 \leq h_{1}(x) \leq h_{2}(x)$ for any $x \in[0, \infty)$, and $0 \geq$ $h_{1}(x) \geq h_{2}(x)$ when $x \in(-\infty, 0)$. For any $\delta>0$, we have that $h_{1}(X) \preceq_{\text {con }}^{-\delta, \delta}$ $h_{2}(X)$.

Roughly speaking, the following corollary reads that a larger "spreading" of the variable leads to a larger variable in the order $\preceq_{c o n}^{-\delta, \delta}$.

Corollary 4 Let $X$ be a random variable. If $\alpha \in(0,1]$, we have that $\alpha X \preceq_{\text {con }}^{-\delta, \delta}$ $X$. If $\alpha \in[1, \infty)$, then $X \preceq_{\text {con }}^{-\delta, \delta} \alpha X$.

Some other properties of condor stochastic orders are analyzed in the following results.

Proposition 11 Let $X$ and $Y$ be random variables such that $X \preceq_{\text {con }}^{p_{2}, p_{3}} Y$. Let $\alpha \in \mathbb{R}$. Then $\alpha X \preceq_{c o n}^{\alpha p_{2}, \alpha p_{3}} \alpha Y$ if $\alpha \geq 0$, and $\alpha X \preceq_{c o n}^{\alpha p_{3}, \alpha p_{2}} \alpha Y$ when $\alpha \leq 0$.

Proposition 12 The order $\preceq_{c o n}^{p_{2}, p_{3}}$ is closed under weak convergence.

Proposition 13 The order $\preceq_{\text {con }}^{p_{2}, p_{3}}$ is closed under mixtures.

The following results reinforce the idea that an investor purchases a condor derivative when he believes that the price of the underlying asset will not be close to the interval of central prices.

Proposition 14 Let $X$ be a random variable and let $\delta>0$ with $X \geq \delta$ a.s. We have that $X \preceq \preceq_{\text {con }}^{-\delta, \delta} X+$ a for any $a \geq 0$.

Proposition 15 Let $X$ be a random variable and let $\delta>0$ with $X \leq-\delta$ a.s. We have that $X \preceq_{\text {con }}^{-\delta, \delta} X+b$ for any $b \leq 0$.

4.3 Connections with other stochastic orders and examples

Next we approach the existence of possible connections of condor stochastic orders with other orders. Some examples of condor orders for some parametric families of distributions are also developed. 
Proposition 16 Let $X$ and $Y$ be random variables such that $X \preceq_{b d} Y$. Let $\delta>0$. Then it holds that $X \preceq \preceq_{\text {con }}^{-\delta, \delta} Y$.

The following example shows that given $\delta>0$, the condor stochastic order $\preceq-\delta, \delta$ is weaker than the bidirectional order.

Example 1 Let $X$ and $Y$ be discrete random variables with $P(X=0)=0.5$, $P(X=1)=0.4, P(X=3)=0.1, P(Y=0)=0.6$ and $P(Y=3)=0.4$. It can be proved that $X \preceq_{b d} Y$ is false. Observe that $X \preceq_{b d} Y$ is satisfied if and only if $F_{X}-F_{Y}$ pivots at 0 (see Proposition 1 in López-Díaz (2010)), that is, if and only if $\left(F_{X}-F_{Y}\right)(x) \leq 0$ when $x<0$ and $\left(F_{X}-F_{Y}\right)(x) \geq 0$ when $x \geq 0$. This condition is not fulfilled. On the other hand, it is not hard to see that $X \preceq_{c o n}^{-2,2} Y$.

The following result states an important connection between the bidirectional order and the family of condor stochastic orders.

Proposition 17 Let $X$ and $Y$ be random variables. Then $X \preceq_{b d} Y$ if and only if $X \preceq$ con $Y$ for any $\delta>0$.

Proposition 16 allows to derive other relations of condor orders with wellknown stochastic orders.

Proposition 18 Let $X$ and $Y$ be random variables with symmetric distributions with respect to their expected values $E X$ and $E Y$ respectively. Let $\delta>0$. If $X \preceq_{\text {peak }} Y$, then $X-E X \preceq_{\text {con }}^{-\delta, \delta} Y-E Y$.

Proposition 19 Let $X$ and $Y$ be random variables such that $X \preceq_{w} Y$. Let $\delta>0$. Then $X-X^{\prime} \preceq$ con $Y-\delta-Y^{\prime}$.

The following result relates condor orders with the increasing concave order and the increasing convex order.

Proposition 20 Let $X$ and $Y$ be random variables such that there exists $\varepsilon>0$ with $X \preceq_{\text {con }}^{-\delta, \delta} Y$ for all $0<\delta<\varepsilon$. If $X$ and $Y$ are negative a.s., then $Y \preceq_{i c x} X$. If $X$ and $Y$ are positive a.s., then $X \preceq_{i c v} Y$.

Corollary 5 Let $X$ and $Y$ be random variables with $E X=E Y$, such that there exists $\varepsilon>0$ with $X \preceq \preceq_{\text {con }}^{-\delta, \delta} Y$ for all $0<\delta<\varepsilon$. If $X$ and $Y$ are negative a.s., or $X$ and $Y$ are positive a.s., we have that $Y \preceq_{c x} X$.

Note that if $X$ and $Y$ are a.s. positive random variables with $X \preceq_{s t} Y$, then $X \preceq$ con $p_{2}, p_{3} \quad Y$ for any values of $p_{2}$ and $p_{3}$ with $p_{2}<p_{3}$ and $p_{2} \leq 0 \leq p_{3}$, since the mappings of $\mathcal{F}_{0}^{p_{2}, p_{3}}$ are increasing in $(0, \infty)$. As a consequence of Proposition $20 \mathrm{ii}$ ), this family of condor stochastic orders is between the usual stochastic order and the increasing concave order.

When $X$ and $Y$ are a.s. negative random variables with $X \preceq s t Y$, then $-Y \preceq_{s t}-X$. By the above paragraph, we obtain that $X \preceq_{i c x} Y$. Thus, under the a.s. negative assumption of random variables, the above family of condor orders is between the usual stochastic order and the increasing convex order. 
Now we analyze some parametric distributions in relation to condor orders. Using Example 1, 2 and 3 in López-Díaz (2010), we obtain conditions for some parametric families to be ordered in condor stochastic orders since they are ordered in the bidirectional stochastic ordering.

Example 2 Let $X \sim_{s t} \exp \left(\lambda_{1}\right)$ and $Y \sim_{s t} \exp \left(\lambda_{2}\right)$. Let $\delta>0$. If $\lambda_{1} \geq \lambda_{2}$ then $X-E X \preceq \preceq_{\text {con }}^{-\delta} Y-E Y$.

Example 3 Given $m \in\{1,2, \ldots\}$ and $\theta \in(0, m]$, let $X^{(m, \theta)}$ be a random variable having a binomial distribution with parameters $m$ and $\theta / m$. For $\theta \in$ $(0,+\infty)$, let $Y^{(\theta)}$ be a random variable with Poisson distribution, $\theta$ being its parameter. Let $\delta>0$. Then $X^{(m, \theta)}-[\theta] \preceq$ con $X^{(m+1, \theta)}-[\theta]$ and $X^{(m, \theta)}-$ $[\theta] \preceq{ }_{\operatorname{con}}^{-\delta, \delta} Y^{(\theta)}-[\theta]$.

Example 4 Let $Z^{(t, \theta)}$ be a random variable having a negative binomial distribution with parameters $t$ and $\theta / t$, where $t \in\{1,2, \ldots\}$ and $\theta \in(0, t]$. Let $\delta>0$. Let $r>t>0$, then there exists an integer $j \geq 1$ such that $Z^{(r, \theta)}-j \preceq \preceq_{c o n}^{-\delta, \delta} Z^{(t, \theta)}-j$, and there exists an integer $j \geq 1$ such that $Y^{(\theta)}-j \preceq$ con $Z^{-\delta, \delta}-j$.

Example 5 Let $X \sim_{s t} N\left(\mu_{X}, \sigma_{X}\right)$ and $Y \sim_{s t} N\left(\mu_{Y}, \sigma_{Y}\right)$. Let $\delta>0$. Then $X-\mu_{X} \preceq_{\operatorname{con}}^{-\delta, \delta} Y-\mu_{Y}$ if and only if $\sigma_{X} \leq \sigma_{Y}$.

Example 6 Let $X \sim_{s t} t_{n}$ and $Y \sim_{s t} t_{m}$ with $m<n$. Let $\delta>0$. Then $X \preceq_{\text {con }}^{-\delta, \delta}$ $Y$.

Example 7 Let $X \sim_{s t} N(0,1), Y \sim_{s t} t_{m}$ and $\delta>0$. Then $X \preceq \preceq_{c o n}^{-\delta, \delta} Y$.

\section{Financial applications}

In economy, prices of assets are sometimes assumed to follow Brownian movements (also known as Brownian motions with drifts) or geometric Brownian movements (also known as Black-Scholes models) (see for instance Dixit and Pindyck (1994), Billingsley (1999), Klebaner (2012), Tretyakov (2013) and Hull (2015)). Let us analyze conditions to order those prices with the condor stochastic orders.

Let $\left(X_{t}\right)_{t \in[0, T]}$ and $\left(Y_{t}\right)_{t \in[0, T]}$ be Brownian movements such that $E X_{t}=$ $r_{X} t, E Y_{t}=r_{Y} t, \operatorname{Var} X_{t}=\sigma_{X}^{2} t$ and $\operatorname{Var} Y_{t}=\sigma_{Y}^{2} t$ for any $t \in[0, T]$, with $r_{X}, r_{Y} \in \mathbb{R}$ and $\sigma_{X}, \sigma_{Y}>0$.

Random variables $X_{t}$ and $Y_{t}$ will represent the prices of two assets at the instant $t \in[0, T]$. We will consider that $t=0$ is the start time of two condor derivatives with the above assets, and $t=T$ stands for the expiration date.

The following results show conditions under which Brownian processes and geometric Brownian processes are ordered in condor stochastic orders at any instant. 
Proposition 21 Let $\left(X_{t}\right)_{t \in[0, T]}$ and $\left(Y_{t}\right)_{t \in[0, T]}$ be Brownian movements under the above conditions. Then $X_{t}-r_{X} t \preceq$ con $Y_{t}^{-\delta, \delta}-r_{Y} t$ for any $t \in[0, T]$ and any $\delta>0$, if and only if $\sigma_{Y} \geq \sigma_{X}$.

By means of this result, we obtain the following proposition for Brownian movements which do not start at zero.

Proposition 22 Let $\left(X_{t}\right)_{t \in[0, T]}$ and $\left(Y_{t}\right)_{t \in[0, T]}$ be Brownian movements under the above conditions. Then $X_{t}-r_{X} t+k \preceq_{c o n}^{k-k+\delta} Y_{t}-r_{Y} t+k$ for any $t \in[0, T]$, any $k \in \mathbb{R}$ and any $\delta>0$, if and only if $\sigma_{Y} \geq \sigma_{X}$.

The above result means that if prices of assets follow Brownian movements, the centered prices plus any constant $k$, are ordered in the condor stochastic order for the put option purchase unit price $k-\delta$ and the call option purchase unit price $k+\delta$, whatever unit prices of the call and put sales.

The following results are in relation to the case in which prices of assets follow the Black-Scholes model. Let $\left(X_{t}\right)_{t \in[0, T]}$ and $\left(Y_{t}\right)_{t \in[0, T]}$ be geometric Brownian movements given by $X_{t}=c\left(e^{r t-\frac{1}{2} \sigma_{X}^{2} t+\sigma_{X} B_{t}}\right)$ and $Y_{t}=$ $c\left(e^{r t-\frac{1}{2} \sigma_{Y}^{2} t+\sigma_{Y} \widetilde{B}_{t}}\right)$, where $\left(B_{t}\right)_{t \in[0, T]}$ and $\left(\widetilde{B}_{t}\right)_{t \in[0, T]}$ are Brownian movements satisfying that $B_{t} \sim_{s t} N(0, \sqrt{t})$ and $\widetilde{B}_{t} \sim_{s t} N(0, \sqrt{t})$ for any $t \in[0, T]$, and $\sigma_{X}, \sigma_{Y}, c, r>0$. Note that $E X_{t}=c e^{r t}=E Y_{t}$ for any $t \in[0, T]$.

Proposition 23 Under the above conditions, consider the processes $\left(X_{t}^{\prime}\right)_{t \in[0, T]}$ and $\left(Y_{t}^{\prime}\right)_{t \in[0, T]}$, given by $X_{t}^{\prime}=X_{t}-c e^{r t}$ and $Y_{t}^{\prime}=Y_{t}-c e^{r t}$ for any $t \in[0, T]$. Let $\delta>0$ and $t \in(0, T]$. Then $X_{t}^{\prime} \preceq_{\text {con }}^{-\delta, \delta} Y_{t}^{\prime}$ if and only if $\sigma_{Y} \geq \sigma_{X}$ and $\delta \geq c e^{r t}\left(e^{\frac{t}{2} \sigma_{X} \sigma_{Y}}-1\right)$.

Proposition 24 Let $\left(X_{t}^{\prime}\right)_{t \in[0, T]}$ and $\left(Y_{t}^{\prime}\right)_{t \in[0, T]}$ be the above processes. Let $\delta>0, p_{2} \in \mathbb{R}$ and $t \in(0, T]$. Then $X_{t}^{\prime}+k \preceq_{\text {con }}^{k-\delta+\delta} Y_{t}^{\prime}+k$ if and only if $\sigma_{Y} \geq \sigma_{X}$ and $\delta \geq c e^{r t}\left(e^{\frac{t}{2} \sigma_{X} \sigma_{Y}}-1\right)$.

\section{An empirical analysis of the condor stochastic order for the DOWJONES and EUROSTOXX indexes}

Next we show how to obtain evidence of a condor stochastic order from an empirical point of view, by means of the results developed in the manuscript.

For that purpose, we use the well-known DOWJONES and EUROSTOXX indexes. Recall that those indexes are the main American and European stock market indexes. The prices of them during the period 2014-2016 were taken from INVERTIA (http://www.invertia.com/). ${ }^{1}$

In order to reduce the position influence, our data consist of the first differences of the indexes. Let us denote the corresponding variables by DOWJONESVAR and EUROSTOXXVAR. The economic interpretation of condors based

\footnotetext{
1 See the web addresses http://www.invertia.com/mercados/bolsa/indices/eurostoxx50/historico-ib020stoxx50 and http://www.invertia.com/mercados/bolsa/indices/dowjones/historico-ib016indu for DOWJONES and EUROSTOXX indexes respectively.
} 
on those differences is the following. Consider $\left(X_{t}\right)$ the stochastic process given by an index, and the first differences $D X_{t}=X_{t}-X_{t-1}$. It holds that $E\left(f_{p_{1},-\delta, \delta, p_{4}, 0}\left(D X_{t}\right)\right)=E\left(f_{p_{1}+X_{t-1},-\delta+X_{t-1}, \delta+X_{t-1}, p_{4}+X_{t-1}, 0}\left(X_{t}\right)\right)$. Therefore condors based on $D X_{t}$ are the same as condors based on $X_{t}$ "centered" in $X_{t-1}$. Figure 2 shows the evolution of those variables.

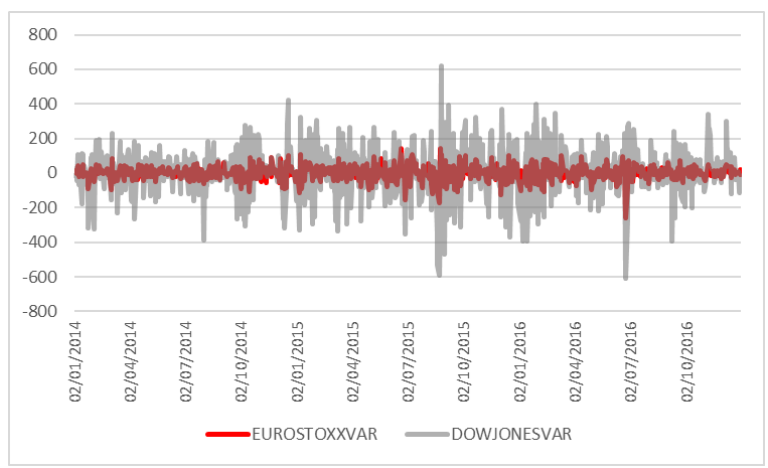

Fig 2 Evolution of the variables EUROSTOXXVAR (red color) and DOWJONESVAR (grey color). Period 2014-2016 in the horizontal axis, values of the variables in the vertical axis

We have used Proposition 3 for our empirical analysis. Note that under the hypothesis of efficient markets, the variations should be independent observations (the first partial autocorrelations are not significative). Normality and non-stationarity of the differences were rejected with Kolmogorov-Smirnov test and Dickey-Fuller test, respectively. The value of $\delta$ was 50 , that is, we consider condor derivatives in which the difference of the strike prices of the call and put purchases is 100 (other differences could be considered). We have depicted the integrals involved in such a result for the corresponding empirical distributions. Namely, we have calculated $\int_{50}^{50+t} \widetilde{F}(x) d x$ for different values of $t$, which ranges from 0 to 950 , with a step of 5 (the value 1000 is a bound from above of the samples), and $\int_{-50+t}^{-50} \widetilde{F}(x) d x$, where $t$ ranges from -950 to 0 , with a step of 5 (-1000 is a bound from below of the samples), and $\widetilde{F}$ stands for an empirical distribution function. The graphical representation was depicted by linear interpolation. The representations are given in Figure 3 and Figure 4. Figure 3 contains the representation of $\int_{50}^{50+t} \widetilde{F}(x) d x$ for the different values of $t$, Figure 4 includes the representation of $\int_{-50+t}^{-50} \widetilde{F}(x) d x$ for the corresponding values of $t$.

The graphical representations show a reasonable evidence that the variable EUROSTOXXVAR is less than DOWJONESVAR in the considered condor order. Therefore the price of a condor derivative based on DOWJONESVAR should be higher (at least the same) than the price of a condor derivative associated with EUROSTOXXVAR, when the difference of strike prices of 


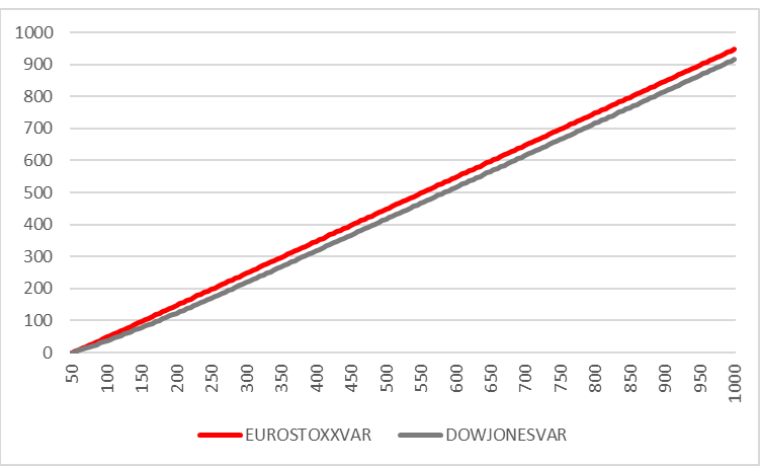

Fig 3 Graphical representation of $\int_{50}^{50+t} \widetilde{F}(x) d x$. Values of $t+50$ in the horizontal axis, values of the integral in the vertical axis. Red color for EUROSTOXXVAR and grey color for DOWJONESVAR

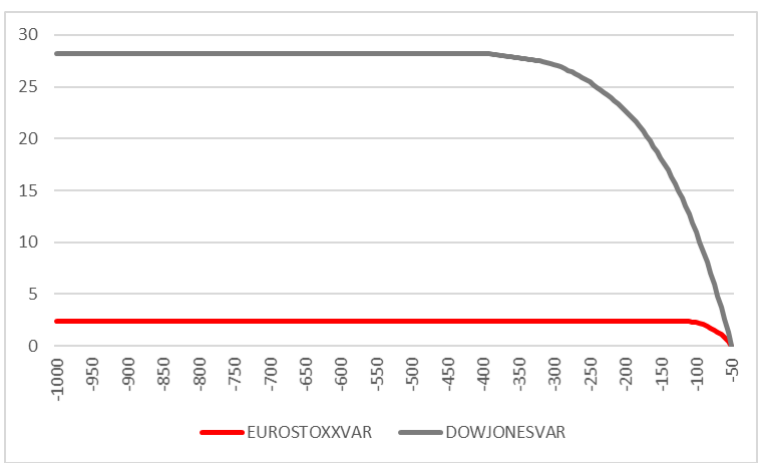

Fig 4 Graphical representation of $\int_{-50+t}^{-50} \widetilde{F}(x) d x$. Values of $-50+t$ in the horizontal axis, values of the integral in the vertical axis. Red color for EUROSTOXXVAR and grey color for DOWJONESVAR

the put and call purchases is 100, whatever unit prices of the put and call sales. Our empirical analysis suggests that a condor derivative based on EUROSTOXXVAR has less expected value than a condor derivative based on the other variable.

\section{Connections between financial derivatives and stochastic orders}

In this manuscript we have introduced and studied in detail a stochastic order to compare investments in condor financial derivatives. Next we show how wellknown stochastic orders are of use to compare investments in other common financial derivatives. We include the definition of those derivatives.

Let $x$ stand for the unit price of an asset of a financial derivative at the expiration date. 
Futures are financial derivatives obligating the buyer to purchase an asset (or the seller to sell an asset) at a predetermined future date and price. The unit benefit of the purchaser of a future derivative is $x-p$, where $p$ is the unit exercise price considered in the contract. Let us denote such a benefit by $F_{p}^{x}$.

A bull call spread is a financial derivative which is constructed by buying a call option with unit strike price $p_{1}$, and selling another call option with unit strike price $p_{2}$, of the same underlying asset and the same expiration date, where $p_{1} \leq p_{2}$. The unit benefit of the buyer of a bull call spread is $\left(x-p_{1}\right)_{+}-k_{1}-\left(x-p_{2}\right)_{+}+k_{2}$, where $k_{1}$ and $k_{2}$ are the corresponding unit premiums. We will denote that benefit by $S_{p_{1}, p_{2}, k_{1}, k_{2}}^{x}$.

A long straddle is a derivative which involves purchasing both a call option and a put option of the same underlying asset, with the same unit exercise price $p$, and the same expiration date. The unit benefit of the purchaser of a long straddle is $(x-p)_{+}-k_{1}+(p-x)_{+}-k_{2}$. It will be denoted by $S T_{p, k_{1}, k_{2}}^{x}$.

Recall that the unit benefit of the buyer of a call option is $(x-p)_{+}-k$. Let $C_{p, k}^{x}$ denote that value.

The following result connects those financial derivatives with well-known stochastic orders like the usual stochastic order, the convex order and the increasing convex order. As in the preceding sections, we will assume the possibility of constructing two derivatives of the same kind with different assets.

Proposition 25 Let $X$ and $Y$ be random variables with finite means, which stand for the unit prices of assets at the expiration date in the above financial derivatives. Then

i) $E X \leq E Y$ if and only if $E F_{p}^{X} \leq E F_{p}^{Y}$ for all $p \in \mathbb{R}$ (comparison of investments in future derivatives),

ii) $X \preceq_{s t} Y$ if and only if $E S_{p_{1}, p_{2}, k_{1}, k_{2}}^{X} \leq E S_{p_{1}, p_{2}, k_{1}, k_{2}}^{Y}$ for all $p_{1}, p_{2}, k_{1}, k_{2} \in$ $\mathbb{R}$ with $p_{1} \leq p_{2}$ (comparison of investments in bull call spreads),

iii) $X \preceq_{i c x} Y$ if and only if $E C_{p, k}^{X} \leq E C_{p, k}^{Y}$ for all $p, k \in \mathbb{R}$ (comparison of investments in call options),

iv) $X \preceq_{c x} Y$ if and only if $E S T_{p, k_{1}, k_{2}}^{X} \leq E S T_{p, k_{1}, k_{2}}^{Y}$ for all $p, k_{1}, k_{2} \in \mathbb{R}$ (comparison of investments in long straddles).

\section{Summary section}

This manuscript shows how the theory of stochastic orders can be used for the comparison of investments in financial derivatives. It is worth noting that some analysts estimate the derivatives market at more than ten times the size of the total world gross domestic product. In this manuscript, a new family of stochastic orders has been introduced to compare investments in condor derivatives. Those orders are useful to reach a decision on how to invest in such derivatives. Characterizations results of the orders have been developed, as well as different properties. By means of those results, conditions to order Brownian processes and geometric Brownian processes in condor stochastic orders have been stated. Theoretical results on the orders have permitted to 
show how to order condor derivatives based on important market indexes from an empirical point of view. In order to reinforce the importance of stochastic orders to compare derivatives, we have shown how other orderings, like the usual stochastic order, the convex order and the increasing convex order, can be used to compare investments in other financial derivatives.

\section{Appendix}

Proofs of mathematical results are included in this appendix.

Proof of Lemma 1. Observe that $f_{p_{2}, p_{2}, p_{3}, p_{4}, k}=f_{p_{2}, p_{2}, p_{3}, p_{4}, 0}+k$ for any $p_{1}, p_{2}, p_{3}, p_{4}, k \in \mathbb{R}$ with $p_{1}<p_{2}<p_{3}<p_{4}$, which leads to the result. Note that $\mathcal{F}_{0}^{p_{2}, p_{3}} \subset \mathcal{F}^{p_{2}, p_{3}}$.

Proof of Lemma 2. Let us see $i$ ). Consider $\left\{f_{p_{2}-1 / n, p_{2}, p_{3}, p_{4}, k}\right\}_{n} \subset \mathcal{F}^{p_{2}, p_{3}}$. Condition $X \preceq_{\text {con }}^{p_{2}, p_{3}} Y$ implies $E\left(f_{p_{2}-1 / n, p_{2}, p_{3}, p_{4}, k}(X)\right) \leq E\left(f_{p_{2}-1 / n, p_{2}, p_{3}, p_{4}, k}(Y)\right)$ for all $n \in \mathbb{N}$. Note that for any $x \in \mathbb{R}$ we have that $\lim _{n} f_{p_{2}-1 / n, p_{2}, p_{3}, p_{4}, k}(x)=$ $f_{p_{2}, p_{2}, p_{3}, p_{4}, k}(x)$, and the above sequence of mappings is uniformly bounded. The Dominated Convergence Theorem leads to $i$ ). In a similar way it is possible to prove $i i$ ) and $i i i)$.

Proof of Proposition 1. Let us suppose that $X \preceq \preceq_{\text {con }}^{p_{2}, p_{3}} Y$. Let $f \in \mathcal{F}_{0}^{-\mu / 2, \mu / 2}$. Note that this class of mapping is a generator of $\preceq_{c o n}^{-\mu / 2, \mu / 2}$ as Lemma 1 reads. Let $T: \mathbb{R} \rightarrow \mathbb{R}$ with $T(x)=x-\left(p_{2}+p_{3}\right) / 2$. Observe that $P_{X} \circ T^{-1}(B)=$ $P_{X-\left(p_{2}+p_{3}\right) / 2}(B)$ for any $B \in \mathcal{B}$. By a change of variable (see for instance Halmos (1950)), we have that

$$
\int_{\mathbb{R}} f(x) d P_{X-\left(p_{2}+p_{3}\right) / 2}=\int_{\mathbb{R}} f \circ T(x) d P_{X}=\int_{\mathbb{R}} f\left(x-\left(p_{2}+p_{3}\right) / 2\right) d P_{X} .
$$

It is not hard to prove that the map $x \rightarrow f\left(x-\left(p_{2}+p_{3}\right) / 2\right)$ belongs to the class $\mathcal{F}_{0}^{p_{2}, p_{3}}$. In fact, if $f=f_{p_{1},-\mu / 2, \mu / 2, p_{4}, 0}$ for some $p_{1}<-\mu / 2$ and $p_{4}>\mu / 2$, then $f\left(x-\left(p_{2}+p_{2}\right) / 2\right)=f_{p_{1}+\left(p_{2}+p_{3}\right) / 2, p_{2}, p_{3}, p_{4}+\left(p_{2}+p_{3}\right) / 2}(x)$. Since $X \preceq_{\text {con }}^{p_{2}, p_{3}} Y$, we obtain that

$\int_{\mathbb{R}} f\left(x-\left(p_{2}+p_{3}\right) / 2\right) d P_{X} \leq \int_{\mathbb{R}} f\left(x-\left(p_{2}+p_{3}\right) / 2\right) d P_{Y}=\int_{\mathbb{R}} f d P_{Y-\left(p_{2}+p_{3}\right) / 2}$,

and so $X-\left(p_{2}+p_{3}\right) / 2 \preceq \preceq_{c o n}^{-\mu / 2, \mu / 2} Y-\left(p_{2}+p_{3}\right) / 2$. The converse can be proved in a similar way.

Proof of Proposition 2. Assume that the condition $X \preceq_{c o n}^{-\delta, \delta} Y$ is satisfied.

Let $t>0$. Take the mapping $f_{-\delta,-\delta, \delta, \delta+t, 0}$, that is, $f_{-\delta,-\delta, \delta, \delta+t, 0}(x)=$ $(x-\delta) I_{(\delta, \delta+t]}(x)+t I_{(\delta+t,+\infty)}(x)$ for any $x \in \mathbb{R}$.

By Lemma 2, $E\left(f_{-\delta,-\delta, \delta, \delta+t, 0}(X)\right) \leq E\left(f_{-\delta,-\delta, \delta, \delta+t, 0}(Y)\right)$ holds. Note that

$$
\int_{\mathbb{R}} f_{-\delta,-\delta, \delta, \delta+t, 0}(x) d P_{X}=\int_{\mathbb{R}}(x-\delta) I_{(\delta, \delta+t]}(x) d P_{X}+\int_{\mathbb{R}} t I_{(\delta+t,+\infty)}(x) d P_{X}
$$




$$
\begin{aligned}
& =\int_{\mathbb{R}} x I_{(\delta, \delta+t]}(x) d P_{X}-\delta P_{X}((\delta, \delta+t])+t P_{X}((\delta+t,+\infty)) \\
= & E\left(X I_{(\delta, \delta+t]}(X)\right)-\delta\left(F_{X}(\delta+t)-F_{X}(\delta)\right)+t\left(1-F_{X}(\delta+t)\right),
\end{aligned}
$$

which implies $i)$.

Let $t<0$. Take the mapping $f_{-\delta+t,-\delta, \delta, \delta, 0}$. That mapping is given by $f_{-\delta+t,-\delta, \delta, \delta, 0}(x)=|t| I_{(-\infty,-\delta+t]}(x)+(-\delta-x) I_{(-\delta+t,-\delta]}(x)$ for any $x \in \mathbb{R}$.

By Lemma $2, E\left(f_{-\delta+t,-\delta, \delta, \delta, 0}(X)\right) \leq E\left(f_{-\delta+t,-\delta, \delta, \delta, 0}(Y)\right)$. Now

$$
\begin{gathered}
\int_{\mathbb{R}} f_{-\delta+t,-\delta, \delta, \delta, 0}(x) d P_{X}=\int_{\mathbb{R}}|t| I_{(-\infty,-\delta+t]}(x) d P_{X}+\int_{\mathbb{R}}(-\delta-x) I_{(-\delta+t,-\delta]}(x) d P_{X} \\
=|t| P_{X}((-\infty,-\delta+t])-\delta P_{X}((-\delta+t,-\delta])-\int_{\mathbb{R}} x I_{(-\delta+t,-\delta]}(x) d P_{X} \\
=|t| F_{X}(-\delta+t)-\delta\left(F_{X}(-\delta)-F_{X}(-\delta+t)\right)-E\left(X I_{(-\delta+t,-\delta]}(X)\right) \\
=|t| F_{X}(-\delta+t)-\delta\left(F_{X}(-\delta)-F_{X}(-\delta+t)\right)+E\left(|X| I_{(-\delta+t,-\delta]}(X)\right),
\end{gathered}
$$

what leads to $i$ ).

Now suppose that $i)$ and $i i)$ hold. Let $f_{p_{1},-\delta, \delta, p_{4}, 0} \in \mathcal{F}_{0}^{-\delta, \delta}\left(p_{1}<-\delta, p_{4}>\right.$ $\delta)$. Note that $f_{p_{1},-\delta, \delta, p_{4}, 0}=f_{\left(p_{1}+\delta\right)-\delta,-\delta, \delta, \delta, 0}+f_{-\delta,-\delta, \delta, \delta+\left(p_{4}-\delta\right), 0}$. Conditions i) and $i$ ), jointly with formulas (2) and (3), imply that $E\left(f_{p_{1},-\delta, \delta, p_{4}, 0}(X)\right) \leq$ $E\left(f_{p_{1},-\delta, \delta, p_{4}, 0}(Y)\right)$, and so $X \preceq \preceq_{\text {con }}^{-\delta, \delta} Y$.

Proof of Proposition 3. Let $t>0$. We have that

$$
\begin{gathered}
E\left(X I_{(\delta, \delta+t]}(X)\right)=\int_{(0,+\infty)} P\left(X I_{(\delta, \delta+t]}(X)>x\right) d x \\
=\int_{(0,+\infty)} P(X \in(\delta, \delta+t], X>x) d x=\int_{(0, \delta+t]} P(X \in(\delta, \delta+t], X>x) d x \\
=\int_{(0, \delta]} P(X \in(\delta, \delta+t]) d x+\int_{(\delta, \delta+t]} P(x<X \leq \delta+t) d x \\
=\delta\left(F_{X}(\delta+t)-F_{X}(\delta)\right)+t F_{X}(\delta+t)-\int_{(\delta, \delta+t]} F_{X}(x) d x
\end{gathered}
$$

Thus, formula $i$ ) of Proposition 2 is equivalently to

$$
\int_{\delta}^{\delta+t} F_{X}(x) d x \geq \int_{\delta}^{\delta+t} F_{Y}(x) d x
$$

for any $t>0$.

On the other hand, if $t<0$ then

$$
\begin{gathered}
E\left(|X| I_{(-\delta+t,-\delta]}(X)\right)=\int_{(0,+\infty)} P\left(|X| I_{(-\delta+t,-\delta]}(X) \geq x\right) d x \\
=\int_{(0,+\infty)} P(X \in(-\delta+t,-\delta],|X| \geq x) d x
\end{gathered}
$$




$$
\begin{gathered}
=\int_{(0, \delta-t]} P(X \in(-\delta+t,-\delta], X \leq-x) d x \\
=\int_{(0, \delta]} P(X \in(-\delta+t,-\delta]) d x+\int_{(\delta, \delta-t]} P(X \in(-\delta+t,-x]) d x \\
=\delta\left(F_{X}(-\delta)-F_{X}(-\delta+t)\right)+\int_{(\delta, \delta-t]} F_{X}(-x) d x+t F_{X}(-\delta+t) .
\end{gathered}
$$

As a consequence, condition $i$ ) in Proposition 2 is the same as

$$
\int_{-\delta+t}^{-\delta} F_{X}(x) d x \leq \int_{-\delta+t}^{-\delta} F_{Y}(x) d x
$$

for any $t<0$.

Proof of Proposition 4. Observe that for any random variable $W, F_{W_{+}}(x)=$ $F_{W}(x) I_{[0, \infty)}(x)$ and $F_{W_{-}}(x)=\left(1-F_{W}\left(-x^{-}\right)\right) I_{[0, \infty)}(x)$.

When $t>0$ we have that

$$
\int_{\delta}^{\delta+t} F_{X}(x) d x=\int_{0}^{t} F_{X}(x+\delta) d x=\int_{0}^{t} F_{X-\delta}(x) d x=\int_{-\infty}^{t} F_{(X-\delta)_{+}}(x) d x
$$

and then condition $i$ ) of Proposition 3 is the same as

$$
\int_{-\infty}^{t} F_{(X-\delta)_{+}}(x) d x \geq \int_{-\infty}^{t} F_{(Y-\delta)_{+}}(x) d x
$$

for any $t \in \mathbb{R}$. By means of Theorem 4.A.2 in Shaked and Shanthikumar (2007), this is equivalent to $(X-\delta)_{+} \preceq_{i c v}(Y-\delta)_{+}$.

When $t<0$ we have that

$$
\int_{-\delta+t}^{-\delta} F_{X}(x) d x=\int_{t}^{0} F_{X}(x-\delta) d x .
$$

Therefore condition $i$ ) of Proposition 3 can be re-written as

$$
\begin{gathered}
t+\int_{t}^{0} F_{X}(x-\delta) d x \leq t+\int_{t}^{0} F_{Y}(x-\delta) d x, \text { that is, } \\
\int_{t}^{0}\left(1-F_{X+\delta}(x)\right) d x \geq \int_{t}^{0}\left(1-F_{Y+\delta}(x)\right) d x .
\end{gathered}
$$

The set of discontinuity points of an increasing map is at most countable, then the above inequality is the same as

$$
\begin{aligned}
& \int_{t}^{0}\left(1-F_{X+\delta}\left(x^{-}\right)\right) d x \geq \int_{t}^{0}\left(1-F_{Y+\delta}\left(x^{-}\right)\right) d x, \text { equivalently, } \\
& \int_{0}^{-t}\left(1-F_{X+\delta}\left(-x^{-}\right)\right) d x \geq \int_{0}^{-t}\left(1-F_{Y+\delta}\left(-x^{-}\right)\right) d x, \text { that is, }
\end{aligned}
$$




$$
\int_{0}^{-t} F_{(X+\delta)_{-}}(x) d x \geq \int_{0}^{-t} F_{(Y+\delta)_{-}}(x) d x
$$

for any $t<0$, or

$$
\int_{0}^{t} F_{(X+\delta)_{-}}(x) d x \geq \int_{0}^{t} F_{(Y+\delta)_{-}}(x) d x
$$

for any $t>0$. It is clear that this is the same as

$$
\int_{-\infty}^{t} F_{(X+\delta)_{-}}(x) d x \geq \int_{-\infty}^{t} F_{(Y+\delta)_{-}}(x) d x
$$

for any $t \in \mathbb{R}$. By Theorem 4.A.2 in Shaked and Shanthikumar (2007), this is $(X+\delta)_{-} \preceq_{i c v}(Y+\delta)_{-}$.

Proof of Proposition 5. Assume that $X \preceq_{\text {con }}^{p_{2}, p_{3}} \quad Y$. Let $f \in \mathcal{F}^{-p_{3},-p_{2}}$. Consider $T: \mathbb{R} \rightarrow \mathbb{R}$ with $T(x)=-x$ for any $x \in \mathbb{R}$. Note that $P_{X} \circ T^{-1}=P_{-X}$. By a change of variable

$$
\int_{\mathbb{R}} f(x) d P_{-X}=\int_{\mathbb{R}} f(-x) d P_{X}
$$

It is not hard to prove that if $f \in \mathcal{F}^{-p_{3},-p_{2}}$, then the mapping $x \rightarrow f(-x)$ belongs to $\mathcal{F}^{p_{2}, p_{3}}$, and so

$$
\int_{\mathbb{R}} f(-x) d P_{X} \leq \int_{\mathbb{R}} f(-x) d P_{Y}=\int_{\mathbb{R}} f(x) d P_{-Y}
$$

which proves that $-X \preceq \preceq_{c o n}^{-p_{3},-p_{2}}-Y$. The converse is implied by the proved part.

Proof of Proposition 6. Let $T: \mathbb{R} \rightarrow \mathbb{R}$ with $T(x)=|x|$. Let $f \in \mathcal{F}_{0}^{-\delta, \delta}$. A change of variable, the symmetry of the distribution and $f(0)=0$, imply that

$$
\begin{gathered}
\int_{\mathbb{R}} f(x) d P_{|X|}=\int_{\mathbb{R}} f(|x|) d P_{X}=\int_{(-\infty, 0)} f(-x) d P_{X}+\int_{(0,+\infty)} f(x) d P_{X} \\
=2 \int_{(0,+\infty)} f(x) d P_{X} .
\end{gathered}
$$

Assume that $X \preceq_{c o n}^{-\delta, \delta} Y$. Let $f \in \mathcal{F}_{0}^{-\delta, \delta}$, thus $f=f_{p_{1},-\delta, \delta, p_{4}, 0}$ for some $p_{1}$ and $p_{4}$ with $p_{1}<-\delta$ and $p_{4}>\delta$. Note that $f I_{(0, \infty)}=f_{-\delta,-\delta, \delta, p_{4}, 0}$. Then

$$
\begin{aligned}
& \int_{\mathbb{R}} f(x) d P_{|X|}=2 \int_{(0,+\infty)} f(x) d P_{X}=2 \int_{(0,+\infty)} f_{-\delta,-\delta, \delta, p_{4}, 0}(x) d P_{X} \\
= & 2 \int_{\mathbb{R}} f_{-\delta,-\delta, \delta, p_{4}, 0}(x) d P_{X} \leq 2 \int_{\mathbb{R}} f_{-\delta,-\delta, \delta, p_{4}, 0}(x) d P_{Y}=\int_{\mathbb{R}} f(x) d P_{|Y|} .
\end{aligned}
$$

Therefore we obtain that $|X| \preceq_{\text {con }}^{-\delta, \delta}|Y|$. 
Now let us suppose that $|X| \preceq_{c o n}^{-\delta, \delta}|Y|$. Let $f \in \mathcal{F}_{0}^{-\delta, \delta}$. Note that the mapping $x \mapsto f(-x)$ belongs to $\mathcal{F}_{0}^{-\delta, \delta}$. Thus

$$
\begin{aligned}
& \int_{\mathbb{R}} f d P_{X}=\int_{(0,+\infty)} f(x) d P_{X}+\int_{(0,+\infty)} f(-x) d P_{X}=\frac{1}{2} \int_{\mathbb{R}} f(x) d P_{|X|} \\
& +\frac{1}{2} \int_{\mathbb{R}} f(-x) d P_{|X|} \leq \frac{1}{2} \int_{\mathbb{R}} f(x) d P_{|Y|}+\frac{1}{2} \int_{\mathbb{R}} f(-x) d P_{|Y|}=\int_{\mathbb{R}} f d P_{Y},
\end{aligned}
$$

which concludes the result.

Proof of Corollary 3. It follows from Propositions 6 and 4.

Proof of Proposition 7. By Proposition 3 we have that

i) $\int_{\delta}^{\delta+t} F_{X}(x) d x=\int_{\delta}^{\delta+t} F_{Y}(x) d x$ for any $t>0$, and

ii) $\int_{-\delta+t}^{-\delta} F_{X}(x) d x=\int_{-\delta+t}^{-\delta} F_{Y}(x) d x$ for any $t<0$.

By the First Fundamental Theorem of Calculus we obtain that $F_{X}(\delta+t)=$ $F_{Y}(\delta+t)$ for all $t>0$ such that $\delta+t$ is a continuity point of $F_{X}$ and $F_{Y}$. Using the right continuity of distributions functions and the density of the set of continuity points of both functions, we obtain that $F_{X}(x)=F_{Y}(x)$ for any $x \geq \delta$. The same reasoning applied to condition $i i)$, provides that $F_{X}(x)=F_{Y}(x)$ for any $x \leq-\delta$.

Proof of Proposition 8. The condition $S^{-}\left(f_{Y}-f_{X}\right)=2$ with sign sequence ,,+-+ , implies that $S^{-}\left(F_{Y}-F_{X}\right)=1$ with sign sequence,+- (see the proof of Theorem 3.A.44 in Shaked and Shanthikumar (2007)). The symmetry of the distributions implies that the crossing point is 0. Moreover, $F_{|X|}(x)=$ $\left(2 F_{X}-2\right) I_{[0, \infty)}(x)$. Therefore $S^{-}\left(F_{|Y|}-F_{|X|}\right)=0$ and $F_{|Y|} \leq F_{|X|}$, which is the same as $|X| \preceq_{s t}|Y|$. Since the mapping $g_{\delta, t}: \mathbb{R} \rightarrow \mathbb{R}$ with $g_{\delta, t}(x)=$ $(x-\delta)_{+}$is increasing, we conclude that $(|X|-\delta)_{+} \preceq s t(|Y|-\delta)_{+}$. That implies $(|X|-\delta)_{+} \preceq_{i c v}(|Y|-\delta)_{+}$. Now Proposition 4 ensures that $|X| \preceq_{\text {con }}^{-\delta, \delta}|Y|$, and Proposition 6 proves the result.

Proof of Proposition 9. Let us see $i)$. We have $F_{(X-\delta)_{+}}(x)=F_{X-\delta}(x) I_{[0, \infty)}(x)=$ $F_{X}(x+\delta) I_{[0,+\infty)}(x)$ for any $x \in \mathbb{R}$, and the same formula holds for the random variable $Y$. Thus if $S^{-}\left(F_{Y}-F_{X}\right) \leq 1$, we obtain that $S^{-}\left(F_{(Y-\delta)_{+}}-F_{(X-\delta)_{+}}\right) \leq$ 1 , and if the second number of sign changes is 1 , so is the first number of sign changes, the sequence of signs being equal. Applying Theorem 4.A.22 (b) in Shaked and Shanthikumar $(2007)$, we conclude that $(X-\delta)_{+} \preceq_{i c v}(Y-\delta)_{+}$.

On the other hand $F_{(X+\delta)_{-}}(x)=\left(1-F_{(X+\delta)}\left(-x^{-}\right)\right) I_{[0, \infty)}(x)=(1-$ $\left.F_{X}\left((-\delta-x)^{-}\right)\right) I_{[0, \infty)}(x)$. As a consequence, we obtain that $S^{-}\left(F_{(Y+\delta)_{-}-}\right.$ $\left.F_{(X+\delta)_{-}}\right) \leq S^{-}\left(F_{Y}-F_{X}\right)$, and if $S^{-}\left(F_{(Y+\delta)_{-}}-F_{(X+\delta)_{-}}\right)=1$ so is $S^{-}\left(F_{Y}-\right.$ $\left.F_{X}\right)=1$, sharing the same sequence of signs. By Theorem 4.A.22 (b) in Shaked and Shanthikumar $(2007)$, we obtain that $(X+\delta)_{-} \preceq_{i c v}(Y+\delta)_{-}$. Now Proposition 4 implies that $X \preceq_{c o n}^{-\delta, \delta} Y$.

In relation to $i i)$, observe that $F_{X_{(n)}}=F_{X}^{n}$. Therefore $S^{-}\left(F_{Y_{(n)}}-F_{X_{(n)}}\right)=$ $S^{-}\left(F_{Y}-F_{X}\right)$. Moreover, $F_{Y_{(n)}}-F_{X_{(n)}}$ and $F_{Y}-F_{X}$ share the same sign changes. Hence $i i$ ) is obtained by $i$ ). 
Taking into account $F_{X_{(1)}}=1-\left(1-F_{X}\right)^{n}$, statement $\left.i i i\right)$ can be obtained in a similar way.

Proof of Proposition 10. It holds that $P_{X} \circ h_{i}^{-1}$ is equal to $P_{h_{i}(X)}$ with $i=1,2$. Let $f \in \mathcal{F}_{0}^{-\delta, \delta}$. We have that

$$
\begin{gathered}
\int_{\mathbb{R}} f(x) d P_{h_{1}(X)}=\int_{\mathbb{R}} f\left(h_{1}(x)\right) d P_{X} \\
=\int_{[0,+\infty)} f\left(h_{1}(x)\right) d P_{X}+\int_{(-\infty, 0)} f\left(h_{1}(x)\right) d P_{X} .
\end{gathered}
$$

If $x \in[0,+\infty)$, we have that $h_{1}(x) \leq h_{2}(x)$ and $f$ is increasing on that set. On the other hand, if $x \in(-\infty, 0)$, then $h_{1}(x) \geq h_{2}(x)$ and on that subset the mapping $f$ is decreasing. As a consequence

$$
\begin{gathered}
\int_{[0,+\infty)} f\left(h_{1}(x)\right) d P_{X}+\int_{(-\infty, 0)} f\left(h_{1}(x)\right) d P_{X} \\
\leq \int_{[0,+\infty)} f\left(h_{2}(x)\right) d P_{X}+\int_{(-\infty, 0)} f\left(h_{2}(x)\right) d P_{X}=\int_{\mathbb{R}} f(x) d P_{h_{2}(X)},
\end{gathered}
$$

which proves the result.

Proof of Proposition 11. The case $\alpha=0$ is trivial. Let $\alpha>0$. Consider $T$ : $\mathbb{R} \rightarrow \mathbb{R}$ with $T(x)=\alpha x$. It holds that $P_{X} \circ T^{-1}=P_{\alpha X}$. Let $f \in \mathcal{F}_{0}^{\alpha p_{2}, \alpha p_{3}}$. By a change of variable,

$$
\int_{\mathbb{R}} f d P_{\alpha X}=\int_{\mathbb{R}} f(x) d P_{X} \circ T^{-1}=\int_{\mathbb{R}} f(\alpha x) d P_{X} .
$$

If $f \in \mathcal{F}_{0}^{\alpha p_{2}, \alpha p_{3}}$, then $f=f_{p_{1}, \alpha p_{2}, \alpha p_{3}, p_{4}, 0}$ for some $p_{1}<\alpha p_{2}$ and $p_{4}>$ $\alpha p_{3}$. It is not hard to prove that the mapping $x \rightarrow f(\alpha x)$ is the function $\alpha f_{p_{1} / \alpha, p_{2}, p_{3}, p_{4} / \alpha, 0}$, which belongs to the class $\mathcal{F}_{0}^{p_{2}, p_{3}}$. As a consequence

$$
\int_{\mathbb{R}} f(\alpha x) d P_{X} \leq \int_{\mathbb{R}} f(\alpha x) d P_{Y}=\int_{\mathbb{R}} f d P_{\alpha Y} .
$$

Therefore $\alpha X \preceq_{c o n}^{\alpha p_{2}, \alpha p_{3}} \alpha Y$.

Now let $\alpha<0$. By the proven part we conclude that $-\alpha X \preceq \preceq_{\text {con }}^{-\alpha p_{2},-\alpha p_{3}}$ $-\alpha Y$. Applying Proposition 5 we deduce that $\alpha X \preceq_{c o n}^{\alpha p_{3}, \alpha p_{2}} \alpha Y$.

Proof of Proposition 12. Note that the mappings of $\mathcal{F}_{0}^{p_{2}, p_{3}}$ are continuous and bounded.

Proof of Proposition 13. The stochastic order is integral which implies the result (see Theorem 2.4.2 in Müller and Stoyan (2002)).

Proof of Proposition 14. Clearly $X-\delta \preceq_{s t} X-\delta+a$. Then $X-\delta \preceq_{i c v} X-\delta+a$. Observe that $(X-\delta)_{+}=X-\delta$ a.s. and $(X+a-\delta)_{+}=X+a-\delta$ a.s. Moreover, $(X+\delta)_{-}=0=(X+a+\delta)_{-}$a.s. Proposition 4 proves the result. 
Proof of Proposition 15. In accordance with Proposition $5, X \preceq c o, \delta \quad X+$ $b$ is equivalent to $-X \preceq_{c o n}^{-\delta, \delta}-X-b$. Now the result is a consequence of Proposition 14.

Proof of Proposition 16. A generator of the bidirectional order is the set $\mathcal{F}=$ $\{f: \mathbb{R} \rightarrow \mathbb{R} \mid f$ is bounded, increasing in $(0, \infty)$, decreasing in $(-\infty, 0)$ and with minimum at the point 0$\}$ (Proposition 5 in López-Díaz (2010)). Observe that $\mathcal{F}_{0}^{-\delta, \delta} \subset \mathcal{F}$, which leads to the result.

Proof of Proposition 17. By Proposition 16, $X \preceq_{b d} Y$ implies $X \preceq_{c o n}^{-\delta, \delta} Y$ for any $\delta>0$.

Now suppose that $X \preceq_{c o n}^{-\delta, \delta} Y$ for any $\delta>0$. The condition $X \preceq_{b d} Y$ is the same as $F_{X}-F_{Y}$ pivots at 0 , that is, $F_{X}(t)-F_{Y}(t) \geq 0$ for any $t \geq 0$ and $F_{X}(t)-F_{Y}(t) \leq 0$ for any $t<0$. Assume that $X \preceq_{b d} Y$ is false. Therefore there exists $t_{0} \geq 0$ with $F_{X}\left(t_{0}\right)<F_{Y}\left(t_{0}\right)$, or there is $t_{0}<0$ satisfying that $F_{X}\left(t_{0}\right)>F_{Y}\left(t_{0}\right)$. Consider now the first possibility. By the right continuity of distribution functions, we can assume that $t_{0}>0$. For the same reason, there exists $\varepsilon>0$ such that $F_{X}<F_{Y}$ on $\left(t_{0}, t_{0}+\varepsilon\right)$, which is a contradiction with Proposition $3 i$ ). The case of the existence of $t_{0}<0$ with $F_{X}\left(t_{0}\right)>F_{Y}\left(t_{0}\right)$, can be analyzed in the same way, using $i$ i) in Proposition 3. Thus we conclude that $X \preceq_{b d} Y$.

Proof of Proposition 18. We have that $X \preceq_{\text {peak }} Y$ implies that $X-E X \preceq_{b d}$ $Y-E Y$ (Corollary 9 in López-Díaz (2010)). The result follows from Proposition 16 .

Proof of Proposition 19. The relation $X \preceq_{w} Y$ is equivalent to $X-X^{\prime} \preceq_{b d}$ $Y-Y^{\prime}$ (Corollary 10 in López-Díaz (2010)), and so we have the result.

Proof of Proposition 20. The sequences of mappings $\left\{(x-1 / m)_{+}\right\}_{m}$ and $\{(x+$ $\left.1 / m)_{-}\right\}_{m}$ are increasing. By the Monotone Convergence Theorem, $\lim _{m} E((X-$ $\left.1 / m)_{+}\right)=E\left(\lim _{m}(X-1 / m)_{+}\right)=E X_{+}$and $\lim _{m} E\left((X+1 / m)_{-}\right)=E\left(\lim _{m}(X+\right.$ $\left.1 / m)_{-}\right)=E X_{-}$. On the other hand, $\lim _{m}(X-1 / m)_{+}=X_{+}$and $\lim _{m}(X+$ $1 / m)_{-}=X_{-}$in the weak convergence since we have the pointwise convergence. The same results are satisfied by random variable $Y$.

By Proposition 4 in this manuscript, Theorem 1.5.9 in Müller and Stoyan (2002) and the relation between $\preceq_{i c x}$ and $\preceq_{i c v}$, we conclude that $X_{+} \preceq_{i c v} Y_{+}$ and $X_{-} \preceq_{i c v} Y_{-}$.

If $X$ and $Y$ are negative a.s., then $Y_{+}=0=X_{+}$a.s., $X_{-}=-X$ and $Y_{-}=-Y$ a.s., and so we conclude that $Y \preceq_{i c x} X$. When $X$ and $Y$ are positive a.s., $Y_{-}=0=X_{-}$a.s., $X_{+}=X$ and $Y_{+}=Y$ a.s., which implies that $X \preceq_{i c v} Y$.

Proof of Corollary 5. It is a consequence of Proposition 20 and Theorem 1.5.3 in Müller and Stoyan (2002). 
Proof of Example 5. Proposition 19 in López-Díaz (2010) reads that $\sigma_{X} \leq \sigma_{Y}$ implies that $X-\mu_{X} \preceq_{b d} Y-\mu_{Y}$, and as a consequence of Proposition 16 we deduce that $X-\mu_{X} \preceq-\delta, \delta \quad Y-\mu_{Y}$.

Let us see the converse. We have that $X-\mu_{X} \preceq \preceq_{\text {con }}^{-\delta, \delta} Y-\mu_{Y}$. Suppose that $\sigma_{Y}<\sigma_{X}$. By the proven part, we obtain that $Y-\mu_{Y} \preceq_{\text {con }}^{-\delta, \delta} X-\mu_{X}$. Applying Proposition 7 , we deduce that $F_{X-\mu_{X}}(t)=F_{Y-\mu_{Y}}(t)$ for any $t \in$ $(-\infty,-\delta] \cup[\delta,+\infty)$. By Proposition 3 and the First Fundamental Theorem of Calculus, we deduce that the density mappings of $X-\mu_{X}$ and $Y-\mu_{Y}$ are the same on $(-\infty,-\delta] \cup[\delta,+\infty)$, and so $\sigma_{X}=\sigma_{Y}$, which contradicts the assumption $\sigma_{Y}<\sigma_{X}$. Therefore $\sigma_{X} \leq \sigma_{Y}$.

Proof of Example 6. Lemma 1 in Finner et al. (2007) reads that the density mappings of $X$ and $Y$ satisfy that $S^{-}\left(f_{Y}-f_{X}\right)=2$ and the sign sequence is ,,+-+ . Now the result follows from Proposition 8.

Proof of Example 7. Lemma 1 and Theorem 3 in Finner et al. (2007) imply that $S^{-}\left(f_{Y}-f_{X}\right)=2$ and the sign sequence is,,+-+ . Proposition 8 proves the result.

Proof of Proposition 21. The result follows from Example 5, note that $X_{t} \sim_{s t}$ $N\left(r_{X} t, \sigma_{X} \sqrt{t}\right)$ and $Y_{t} \sim_{s t} N\left(r_{Y} t, \sigma_{Y} \sqrt{t}\right)$.

Proof of Proposition 22. Proposition 21 reads that $\sigma_{Y} \geq \sigma_{X}$ if and only if $X_{t}-r_{X} t \preceq{ }_{c o n}^{-\delta, \delta} Y_{t}-r_{Y} t$ for any $t \in[0, T]$ and any $\delta>0$. Now take $p_{2}=k-\delta$ and $p_{3}=k+\delta$ with $k \in \mathbb{R}$ and $\delta>0$ in Proposition 1, which proves the result.

Proof of Proposition 23. We will prove the result by means of Proposition 3.

Let $z \leq-\delta$. We have that $F_{X_{t}^{\prime}}(z)=P\left(X_{t}^{\prime} \leq z\right)=P\left(X_{t} \leq c e^{r t}+z\right)$. Assume that $z$ satisfies that $c e^{r t}+z>0$, that is, $-c e^{r t}<z$, otherwise the above probability is equal to 0 . Take $m_{z}=\ln \left(1+z / c e^{r t}\right)$, thus $c e^{r t}+z=c e^{r t+m_{z}}$. The above probability satisfies that

$$
\begin{gathered}
P\left(X_{t} \leq c e^{r t}+z\right)=P\left(X_{t} \leq c e^{r t+m_{z}}\right)=P\left(-\frac{1}{2} \sigma_{X}^{2} t+\sigma_{X} B_{t} \leq m_{z}\right) \\
=P\left(B_{t} \leq \frac{1}{2} \sigma_{X} t+\frac{m_{z}}{\sigma_{X}}\right)=F_{B_{t}}\left(\frac{1}{2} \sigma_{X} t+\frac{m_{z}}{\sigma_{X}}\right) .
\end{gathered}
$$

The same result holds for the process $\left(Y_{t}^{\prime}\right)_{t \in[0, T]}$.

Suppose that condition $i$ ) in Proposition 3 holds.

Recall that $B_{t} \sim_{s t} \widetilde{B}_{t} \sim_{s t} N(0, \sqrt{t})$. By the continuity of $F_{B_{t}}$ and $F_{\widetilde{B}_{t}}$ we obtain that

$$
\frac{1}{2} \sigma_{X} t+\frac{m_{-\delta}}{\sigma_{X}} \leq \frac{1}{2} \sigma_{Y} t+\frac{m_{-\delta}}{\sigma_{Y}} \text {, that is, } 0 \leq\left(\sigma_{Y}-\sigma_{X}\right)\left(\frac{t}{2}-m_{-\delta} \frac{1}{\sigma_{X} \sigma_{Y}}\right) .
$$

Observe that $m_{-\delta}=\ln \left(1+(-\delta) / c e^{r t}\right)$ is negative, and so $\sigma_{Y} \geq \sigma_{X}$. Conversely, if $\sigma_{Y} \geq \sigma_{X}$ then

$$
F_{B_{t}}\left(\frac{1}{2} \sigma_{X} t+\frac{m_{z}}{\sigma_{X}}\right) \leq F_{\widetilde{B}_{t}}\left(\frac{1}{2} \sigma_{Y} t+\frac{m_{z}}{\sigma_{Y}}\right)
$$


for any $z \leq-\delta$ since $m_{z}$ is negative, and so condition $\left.i i\right)$ in Proposition 3 is satisfied. Therefore statement $i i)$ in Proposition 3 is equivalent to $\sigma_{Y} \geq \sigma_{X}$. Now take $z \geq \delta$. We have that $F_{X_{t}^{\prime}}(z)=P\left(X_{t}^{\prime} \leq z\right)=P\left(X_{t} \leq c e^{r t}+z\right)=$ $P\left(X_{t} \leq c e^{r t+m_{z}}\right)$, where $m_{z}=\ln \left(1+z / c e^{r t}\right)$, note that in this case $m_{z}>0$. Thus

$$
\begin{aligned}
& P\left(X_{t} \leq c e^{r t+m_{z}}\right)=P\left(-\frac{1}{2} \sigma_{X}^{2} t+\sigma_{X} B_{t} \leq m_{z}\right) \\
& =P\left(B_{t} \leq \frac{1}{2} \sigma_{X} t+\frac{m_{z}}{\sigma_{X}}\right)=F_{B_{t}}\left(\frac{1}{2} \sigma_{X} t+\frac{m_{z}}{\sigma_{X}}\right) .
\end{aligned}
$$

Assume that condition $i$ ) in Proposition 3 is satisfied. By the right continuity of distribution functions, we obtain that

$$
\frac{1}{2} \sigma_{X} t+\frac{m_{\delta}}{\sigma_{X}} \geq \frac{1}{2} \sigma_{Y} t+\frac{m_{\delta}}{\sigma_{Y}}, \text { that is, } 0 \geq\left(\sigma_{Y}-\sigma_{X}\right)\left(\frac{t}{2}-\frac{m_{\delta}}{\sigma_{x} \sigma_{Y}}\right) .
$$

Since $\sigma_{Y} \geq \sigma_{X}$ by the first part of the proof, we conclude that $m_{\delta} \geq$ $\frac{t}{2} \sigma_{X} \sigma_{Y}$, that is, $\delta \geq c e^{r t}\left(e^{\frac{t}{2} \sigma_{X} \sigma_{Y}}-1\right)$. Conversely, if $\delta \geq c e^{r t}\left(e^{\frac{t}{2} \sigma_{X} \sigma_{Y}}-1\right)$, that is, $m_{\delta} \geq \frac{t}{2} \sigma_{X} \sigma_{Y}$, then $m_{z} \geq m_{\delta} \geq \frac{t}{2} \sigma_{X} \sigma_{Y}$ for any $z \geq \delta$, and so $F_{B_{t}}\left(\frac{1}{2} \sigma_{X} t+\frac{m_{z}}{\sigma_{X}}\right) \geq F_{\widetilde{B}_{t}}\left(\frac{1}{2} \sigma_{Y} t+\frac{m_{z}}{\sigma_{Y}}\right)$, which concludes $\left.i\right)$ in Proposition 3, and so the proof of the result.

Proof of Proposition 24. It follows applying Proposition 23 and Proposition 1.

Proof of Proposition 25. Statement $i$ ) is clear.

In relation to $i i)$, the real mapping on $\mathbb{R}$ given by $x \rightarrow S_{p_{1}, p_{2}, k_{1}, k_{2}}^{x}$ is increasing for any $p_{1}, p_{2}, k_{1}, k_{2} \in \mathbb{R}$ with $p_{1} \leq p_{2}$. Therefore $X \preceq_{s t}^{p_{1}, p_{2}, k_{1}, k_{2}}$ implies that $E S_{p_{1}, p_{2}, k_{1}, k_{2}}^{X} \leq E S_{p_{1}, p_{2}, k_{1}, k_{2}}^{Y}$ for all $p_{1}, p_{2}, k_{1}, k_{2} \in \mathbb{R}$ with $p_{1} \leq p_{2}$.

Conversely, note that $E S_{p_{1}, p_{2}, k_{1}, k_{2}}^{X}=\pi_{X}\left(p_{1}\right)-\pi_{X}\left(p_{2}\right)+k_{2}-k_{1}$. Thus, $E S_{p_{1}, p_{2}, k_{1}, k_{2}}^{X} \leq E S_{p_{1}, p_{2}, k_{1}, k_{2}}^{Y}$ for all $p_{1}, p_{2}, k_{1}, k_{2} \in \mathbb{R}$ with $p_{1} \leq p_{2}$, implies that $\pi_{X}\left(p_{1}\right)-\pi_{X}\left(p_{2}\right) \leq \pi_{Y}\left(p_{1}\right)-\pi_{Y}\left(p_{2}\right)$. That is $\pi_{Y}-\pi_{X}$ is decreasing. By Theorem 1.5.13 in Müller and Stoyan (2002), we obtain that $X \preceq_{s t} Y$.

Statment iii) follows from Theorem 1.5.7 in Müller and Stoyan (2002).

For the last statement, note that the real mapping on $\mathbb{R}$ defined by $x \rightarrow$ $S T_{p, k_{1}, k_{2}}^{x}$ is convex for any $p, k_{1}, k_{2} \in \mathbb{R}$. Thus $X \preceq_{c x} Y$ leads to $E S T_{p, k_{1}, k_{2}}^{X} \leq$ $E S T_{p, k_{1}, k_{2}}^{Y}$ for all $p, k_{1}, k_{2} \in \mathbb{R}$.

For the converse, take $k_{1}=k_{2}=0$. Now note that $\lim _{p^{\prime} \rightarrow-\infty} \frac{1}{2}\left(S T_{p, 0,0}^{X}+\right.$ $\left.S T_{p^{\prime}, 0,0}^{X}-\left(p-p^{\prime}\right)\right)=C_{p, 0}^{X}$. Since $E\left(S T_{p, 0,0}^{X}+S T_{p^{\prime}, 0,0}^{X}\right) \leq E\left(S T_{p, 0,0}^{Y}+S T_{p^{\prime}, 0,0}^{Y}\right)$ for any $p, p^{\prime} \in \mathbb{R}$, the Dominated Convergence Theorem implies that $E\left(C_{p, 0}^{X}\right) \leq$ $E\left(C_{p, 0}^{Y}\right)$. By Theorem 1.5.7 in Müller and Stoyan (2002), $X \preceq_{i c x} Y$ holds.

On the other hand, since $S T_{p, 0,0}^{X}=(X-p)_{+}+(p-X)_{+}$we obtain that $\lim _{p \rightarrow-\infty} S T_{p, 0,0}^{X}+p=X$ and $\lim _{p \rightarrow+\infty} S T_{p, 0,0}^{X}-p=-X$. By the Dominated Convergence Theorem, we have that $\lim _{p \rightarrow-\infty} E\left(S T_{p, 0,0}^{X}\right)+p=$ $E X$ and $\lim _{p \rightarrow+\infty} E\left(S T_{p, 0,0}^{X}\right)-p=-E X$. Therefore we deduce that $E X=$ $E Y$, which in conjunction with $X \preceq_{i c x} Y$ implies that $X \preceq_{c x} Y$.

Acknowledgements The authors would like to thank the Referees and the Editor for their interesting comments and suggestions which have improved the manuscript. 


\section{References}

Belzunce F, Martínez-Riquelme C, Mulero J (2016) An Introduction to Stochastic Orders. Elsevier/Academic Press, Amsterdam.

Bickel PJ, Lehmann EL (1976) Descriptive statistics for nonparametric models. III. Dispersion. Ann Stat 4:1139-1158.

Billingsley P (1999) Convergence of probability measures. Second edition. Wiley Series in Probability and Statistics. John Wiley \& Sons, Inc., New York.

Birnbaum ZW (1948) On random variables with comparable peakedness. Ann Math Stat 19:76-81.

Cohen G (2005) The Bible of Options Strategies. Pearson Education, Inc., New Jersey.

Dixit AK, Pindyck RS (1994) Investment under Uncertainty. Princeton University Press, Princenton, New Jersey.

Finner H, Roters M, Dickhaus T (2007) Characterizing density crossing points. Am Stat 61:28-33.

Giovagnoli A, Wynn HP (1995) Multivariate dispersion orderings. Stat Probab Lett 22:325332 .

Halmos PR (1950) Measure Theory. D. Van Nostrand Company, Inc., New York.

Hull JC (2015) Options, futures and other derivatives. Pearson, Boston.

Hunt P, Kennedy J (2004) Financial Derivatives in Theory and Practice. Wiley Series in Probability and Statistics. John Wiley \& Sons, Ltd., Chichester.

Jarrow RA, Chatterjea A (2013) An Introduction to Derivative Securities, Financial Markets, and Risk Management. W.W. Norton \& Co, New York.

Klebaner FC (2012) Introduction to stochastic calculus with applications. Third edition. Imperial College Press, London.

Kolb RW, Overdahl JA (2002) Financial Derivatives, Third Edition. John Wiley \& Sons, Inc., New Jersey.

López-Díaz M (2010) A stochastic order for random variables with applications. Aust Nz J Stat 52:1-16.

Müller A (1997) Stochastic orders generated by integrals: a unified study. Adv Appl Probab 29:414-428.

Müller A (1998) Another tale of two tails: on characterizations of comparative risk. J Risk Uncertainty 16:187-197.

Müller A, Stoyan D (2002) Comparison Methods for Stochastic Models and Risks. John Wiley \& Sons, Chichester.

Shaked M, Shanthikumar JG (2007) Stochastic Orders. Springer, New York.

Tretyakov MV (2013) Introductory course on financial mathematics. Imperial College Press, London. 